Tạp chí Khoa học và Công nghệ biển T10 (2010). Số 3. Tr 01 - 13

\title{
TÍNH TOÁN CÔNG TRÌNH NGÀ̀M TRONG NỀN SAN HÔ CHỊU TÁC DỤNG ĐỒNG THỜI CỦA ĐỘNG ĐẤT VÀ LỬC THUỶ TĨNH
}

\author{
NGUYẼ̃N TÁT NGÂN
}

Học viện Kỹ thuật quân sự

\begin{abstract}
Tóm tắt. Bài báo trình bày phưong pháp tính tuoong tác giũa công trình ngầm trong nền san hô chịu tác dụng của tải trọng do động đất và lục thuỷ tĩnh nên. Bài toán được giải quyết bằng mô hình phẳng, trên cơ sở phuoong pháp phần tử hưu hạn (PTHH), kết hợp phần biến dạng phẳng dạng tam giác 3 điểm nút và phần tư tiếp xúc hai chiều $(2 D)$. Với thuật toán kết hợp tích phân trực tiếp Newmark và phuoong pháp lặp Newton-Raphson tác giả lập trình tính toán trong môi truờng Matlab để giải phuoong trình phi tuyến liên kết. Kết quả tính toán số phù hợp quy luật cơ học và toán học cho thấy khả năng của chưong trình đã lập. Nội dung của bài báo có thể làm tài liệu tham khảo cho việc nghiên cứu và tính toán, thiết kế các kết cấu công trình ngầm trong nền san hô.
\end{abstract}

\section{MỞ ĐÀ̀U}

Động đất là hiện tương thiên nhiên đã và sẽ gây ra nhiều thảm hoạ đối với con người và các công trình xây dựng. Từ trước tới nay, con người đã có rất nhiều nỗ lực trong việc nghiên cứu phòng, chống động đất để bảo vệ sinh mạng của mình và tài sản vật chất xã hội. Một trong những hướng nghiên cứu hiện nay là đưa ra các phương pháp tính toán, thiết kế công trình đảm bảo giảm thiểu tác động của động đất, nâng cao hiệu quả sử dụng, tăng tuổi thọ của công trình. Mặc dù đã có những thành công đáng kể trong lĩnh vực này, song thực tế vẫn còn nhiều thảm hoạ do động đất gây ra trên Thế giới hiện nay. Khi động đất xảy ra, do hiện tượng dao động mạnh của nền đất, nên kết cấu cũng bị dao động, do đó tự bản thân kết cấu (hệ kết cấu - nền) cũng chịu tác dụng của lực quán tính do gia tốc chuyển động của nền (gia tốc nền) gây ra. Do vậy, trong tính toán công trình chịu tác dụng của động đất, gia tốc nền là một trong những yếu tố rất quan trọng.

Các công trình trên các đảo san hô xa bờ cũng có khả năng chịu tác động của động đất, khi các công trình này hư hỏng, ngoài sự thiệt hại về vật chất, con người như các công trình trong đất liền thì vấn đề bảo đảm điều kiện tác chiến trên đảo không còn, ảnh hưởng lớn đến khả năng giữ vững chủ quyền trên biển - điều này là một trong những đặc thù của 
các công trình biển, đảo. Vì vậy, nghiên cứu tính toán các công trình ngầm trong nền san hô chịu tác dụng của tải trọng động đất là vấn đề cần thiết.

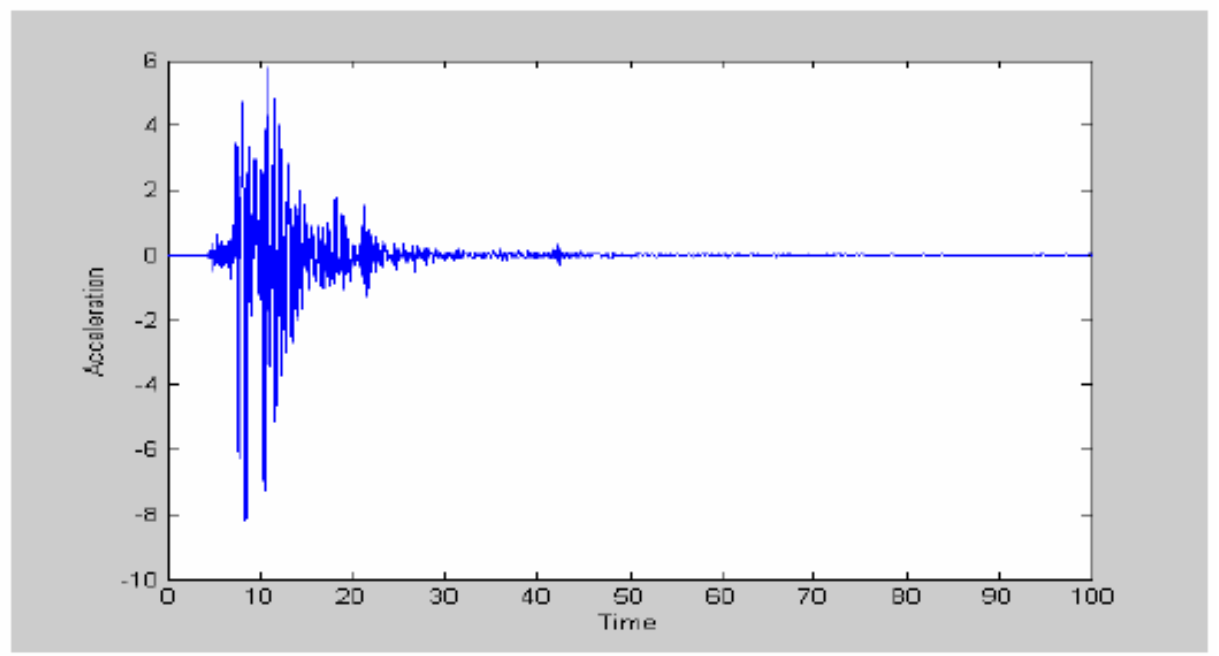

Hình 1: Gia tốc nền trong trận động đất năm 1995 tại Kobe - Nhật Bản [5]

\section{CÁC GIẢ THIẾT, MÔ HÌNH VÀ PHƯƠNG PHÁP TÍNH}

\section{Các giả thiết và mô hình tính của bài toán}

Bài toán được giải quyết dựa vào các giả thiết sau:

- Kết cấu công trình đàn hồi, biến dạng tuyến tính.

- Mỗi lớp nền san hô là vật liệu đồng nhất, đẳng hướng, đàn hồi tuyến tính. Quá trình kết cấu làm việc, không có hiện tượng tách, trượt giữa các lớp nền với nhau.

- Hệ kết cấu công trình và nền làm việc trong điều kiện biến dạng phẳng. Liên kết giữa kết cấu và nền san hô được thay thế bằng liên kết nút giữa các phần tử biến dạng phẳng $2 \mathrm{D}$ thông qua liên kết với phần tử tiếp xúc Goodman $[1,3]$. Liên kết tiếp xúc giữa kết cấu và nền san hô là liên kết một chiều.

- Khi tính toán, đối với vật liệu san hô, bỏ qua lực dính giữa kết cấu và nền.

Theo hướng này, tách từ hệ thực bán vô hạn ra một miền hữu hạn bao gồm kết cấu và một phần nền gọi là miền nghiên cứu, trên biên miền nghiên cứu được đặt các liên kết, việc tính toán được thực hiện trên miền nghiên cứu đã xác định. Kích thước biên của miền nghiên cứu được xác định theo phương pháp lặp [1,7] (hình 2). 


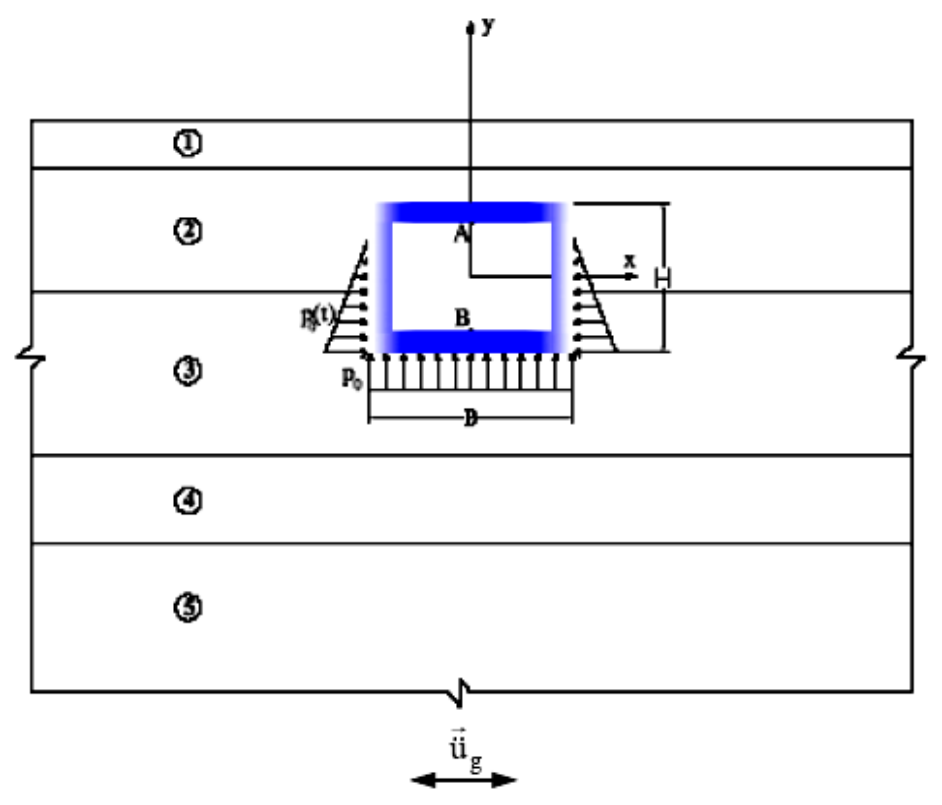

Hình 2: Mô hình thực của bài toán

\section{Phương pháp tính và các loại PTHH}

Để tính toán kết cấu theo mô hình nói trên, tác giả sử dụng phương pháp PTHH, trong đó các loại phần tử sử dụng là: Đối với nền san hô và kết cấu sử dụng loại phần tử tam giác phẳng 3 điểm nút. Đối với lớp tiếp xúc giữa kết cấu và nền san hô sử dụng loại phần tử tiếp xúc tứ giác 4 điểm nút (four nodes slip element).

\section{THIẾT LậP CÁC PHƯƠNG TRÌNH CƠ BẢN BẦNG PHƯƠNG PHÁP PTHH}

\section{Các quan hệ đối với phần tử thuộc nền và kết cấu}

Xét phần tử tam giác phẳng 3 điểm nút, việc đánh số thứ tự nút của phần tử và lựa chọn hệ toạ độ cục bộ của phần tử được quy ước như trên hình 3.

Chuyển vị tại một điểm bất kỳ trong phạm vi phần tử được nội suy từ véc tơ chuyển vị nút của phần tử theo biểu thức [2]:

$$
\{\mathrm{u}\}=[\mathrm{N}]_{\mathrm{m}}\{\mathrm{U}\}_{\mathrm{m}}
$$

trong đó:

$\{\mathrm{u}\}=\left\{\begin{array}{ll}\mathrm{u}_{\mathrm{x}} & \mathrm{u}_{\mathrm{y}}\end{array}\right\}^{\mathrm{T}}-$ véc tơ chuyển vị tại điểm $(\mathrm{x}, \mathrm{y})$ thuộc phần tử,

$[\mathrm{N}]_{\mathrm{m}}$ - ma trận hàm dạng của phần tử thứ $m$, 
$\{\mathrm{U}\}_{\mathrm{m}}$ - véc tơ chuyển vị nút của phần tử thứ $m$, nó có dạng:

$\{\mathrm{U}\}_{\mathrm{m}}=\left\{\begin{array}{llllll}\mathrm{U}_{1 \mathrm{x}} & \mathrm{U}_{1 \mathrm{y}} & \mathrm{U}_{2 \mathrm{x}} & \mathrm{U}_{2 \mathrm{y}} & \mathrm{U}_{3 \mathrm{x}} & \mathrm{U}_{3 \mathrm{y}}\end{array}\right\}^{\mathrm{T}}$

Ma trận hàm dạng của phần tử có dạng:

$$
[\mathrm{N}]_{\mathrm{m}}=\left[\begin{array}{cccccc}
\mathrm{N}_{1} & 0 & \mathrm{~N}_{2} & 0 & \mathrm{~N}_{3} & 0 \\
0 & \mathrm{~N}_{2} & 0 & \mathrm{~N}_{2} & 0 & \mathrm{~N}_{3}
\end{array}\right]
$$

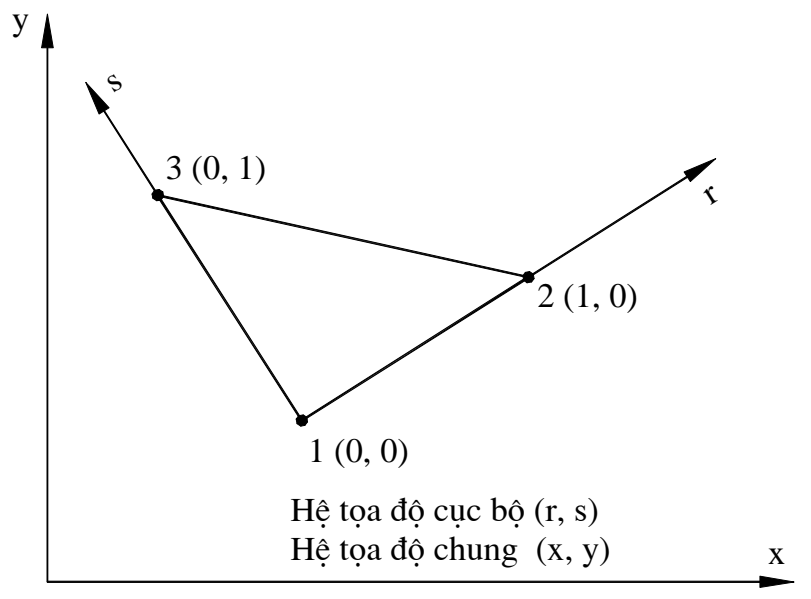

Hình 3: Số hiệu nút của phần tử, hệ toạ độ chung và toạ độ cục bộ của phần tử

Biểu thức hàm dạng của phần tử tam giác viết trong toạ độ cục bộ và tọa độ chung được cho trong bảng 1 .

Bảng 1: Hàm dạng của phần tử tam giác

\begin{tabular}{|c|c|}
\hline Toạ độ cục bộ & Toạ độ chung \\
\hline$N_{1}=1-r-s$ & $N_{1}=\left(a_{1}+b_{1} x+c_{1} y\right) / 2 \Delta$ \\
\hline$N_{2}=r$ & $N_{2}=\left(a_{2}+b_{2} x+c_{2} y\right) / 2 \Delta$ \\
\hline$N_{3}=s$ & $N_{3}=\left(a_{3}+b_{3} x+c_{3} y\right) / 2 \Delta$ \\
\hline
\end{tabular}

trong đó $\Delta=\frac{1}{2}\left|\begin{array}{lll}1 & \mathrm{x}_{1} & \mathrm{y}_{1} \\ 1 & \mathrm{x}_{2} & \mathrm{y}_{2} \\ 1 & \mathrm{x}_{3} & \mathrm{y}_{3}\end{array}\right|$ - là diện tích phần tử,

$$
\begin{aligned}
& \mathrm{a}_{1}=\mathrm{x}_{2} \mathrm{y}_{3}-\mathrm{x}_{3} \mathrm{y}_{2} ; \quad \mathrm{a}_{2}=\mathrm{x}_{3} \mathrm{y}_{1}-\mathrm{x}_{1} \mathrm{y}_{3} ; \quad \mathrm{a}_{3}=\mathrm{x}_{1} \mathrm{y}_{2}-\mathrm{x}_{2} \mathrm{y}_{1} ; \\
& \text { với: } \mathrm{b}_{1}=\mathrm{y}_{2}-\mathrm{y}_{3} ; \quad \mathrm{b}_{2}=\mathrm{y}_{3}-\mathrm{y}_{1} ; \quad \mathrm{b}_{3}=\mathrm{y}_{1}-\mathrm{y}_{2} ; \\
& \mathrm{c}_{1}=\mathrm{x}_{3}-\mathrm{x}_{2} ; \quad \mathrm{c}_{2}=\mathrm{x}_{1}-\mathrm{x}_{3} ; \quad \mathrm{c}_{3}=\mathrm{x}_{2}-\mathrm{x}_{1}
\end{aligned}
$$


Véc tơ biến dạng của phần tử $\{\varepsilon\}$ được viết như sau:

$$
\{\varepsilon\}=[B]_{\mathrm{m}}\{\mathrm{U}\}_{\mathrm{m}}
$$

Ma trận biến dạng-chuyển vị của phần tử $[\mathrm{B}]_{\mathrm{m}}$ được xác định:

$$
[\mathrm{B}]_{\mathrm{m}}=\left[\begin{array}{cccccc}
\frac{\partial \mathrm{N}_{1}}{\partial \mathrm{x}} & 0 & \frac{\partial \mathrm{N}_{2}}{\partial \mathrm{x}} & 0 & \frac{\partial \mathrm{N}_{3}}{\partial \mathrm{x}} & 0 \\
0 & \frac{\partial \mathrm{N}_{1}}{\partial \mathrm{y}} & 0 & \frac{\partial \mathrm{N}_{2}}{\partial \mathrm{y}} & 0 & \frac{\partial \mathrm{N}_{3}}{\partial \mathrm{y}} \\
\frac{\partial \mathrm{N}_{1}}{\partial \mathrm{y}} & \frac{\partial \mathrm{N}_{1}}{\partial \mathrm{x}} & \frac{\partial \mathrm{N}_{2}}{\partial \mathrm{y}} & \frac{\partial \mathrm{N}_{2}}{\partial \mathrm{x}} & \frac{\partial \mathrm{N}_{3}}{\partial \mathrm{y}} & \frac{\partial \mathrm{N}_{3}}{\partial \mathrm{x}}
\end{array}\right]
$$

Ma trận độ cứng phần tử được xác định theo biểu thức sau:

$$
[\mathrm{K}]_{\mathrm{m}}=\int_{\mathrm{A}_{\mathrm{m}}} \mathrm{h}[\mathrm{B}]_{\mathrm{m}}^{\mathrm{T}}[\mathrm{D}][\mathrm{B}]_{\mathrm{m}} \mathrm{dA} \mathrm{m}_{\mathrm{m}}
$$

trong đó: [D] là ma trận vật liệu phần tử, phụ thuộc vào modul Young E, hệ số Possion v.

Sử dụng phương phương pháp tích phân số cầu phương Gauss-Legendre, biểu thức (6) được viết lại: $[\mathrm{K}]_{\mathrm{m}}=\mathrm{h} \sum_{\mathrm{i}=1}^{\mathrm{n}}[\mathrm{B}]_{\mathrm{m}, \mathrm{i}}^{\mathrm{T}}[\mathrm{D}][\mathrm{B}]_{\mathrm{m}, \mathrm{i}} \operatorname{det}\left|\mathrm{J}_{\mathrm{i}}\right| \mathrm{W}_{1 \mathrm{i}} \mathrm{W}_{2 \mathrm{i}}$

trong đó: $i$ - điểm tích phân thứ $i$ có toạ độ $\left(r_{\mathrm{i}}, s_{\mathrm{i}}\right), n$ - tổng số điểm tích phân, det $|\mathrm{J}|$ định thức của ma trận Jacobi, $W_{1 i}, W_{2 i}$ - trọng số của phép tích phân cầu phương, chỉ số $i$ biểu diễn giá trị của hàm tại điểm Gauss thứ $i$.

Ma trận khối lượng của phần tử được xác định theo công thức sau:

$$
[\mathrm{M}]_{\mathrm{m}}=\int_{\mathrm{A}_{\mathrm{m}}} \rho \mathrm{h}[\mathrm{N}]_{\mathrm{m}}^{\mathrm{T}}[\mathrm{N}]_{\mathrm{m}} \mathrm{dA} \mathrm{m}_{\mathrm{m}}
$$

và cũng được tính theo phương pháp tích phân số cầu phương Gauss-Legendre.

trong đó: $\rho$ - khối lượng riêng của vật liệu của phần tử thứ $m ; A_{\mathrm{m}}$ - diện tích phần tử thứ $m$; $h$ - chiều dày phần tử,

\section{Các quan hệ đối với phần tử thuộc lớp tiếp xúc (PTTX)}

Phần tử tiếp xúc 2 chiều được bố trí vào lớp tiếp xúc giữa kết cấu công trình và nền san hô thể hiện tính chất liên kết một chiều của nền. Mô hình phần tử tiếp xúc có chiều dày "bằng không" được chỉ ra như trên hình 4 , do chiều dày $\mathrm{t}$ của phần tử gần như bằng 0 nên các cặp nút 1 và 4,2 và 3,5 và 6 có cùng toạ độ. 


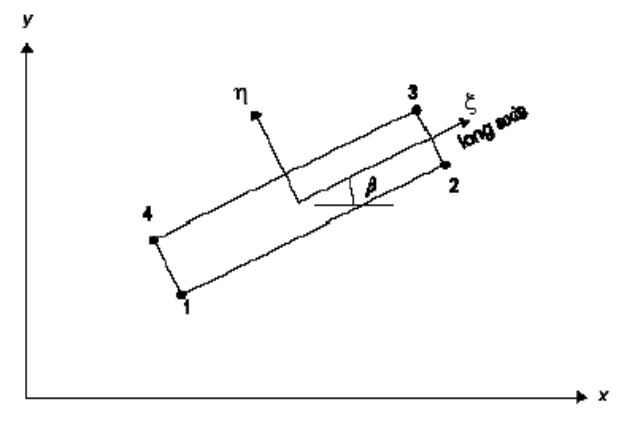

(a)

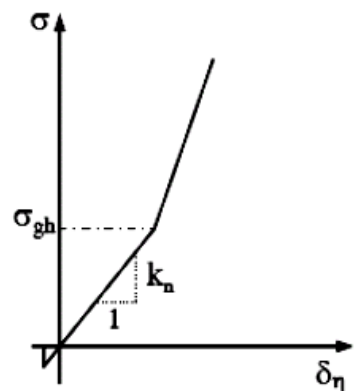

(b)

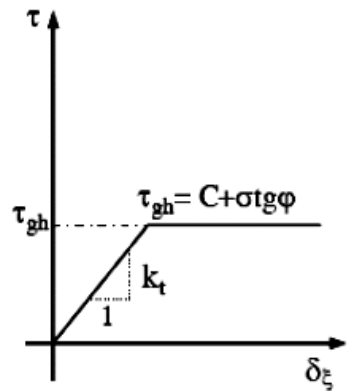

(c)

Hình 4: Mô hình phần tử tiếp xúc Goodman $(1 \div 4$ - các nút). a) Sơ đồ hình học của phần tử tiếp xúc; b) Quan hệ ứng suất pháp tuyến với biến dạng pháp tuyến; c) Quan hệ ứng suất tiếp tuyến với biến dạng tiếp tuyến

Quan hệ giữa số gia ứng suất và số gia biến dạng: $\left\{\begin{array}{l}\Delta \sigma \\ \Delta \tau\end{array}\right\}=\left[\mathrm{D}_{\mathrm{se}}\right]\left\{\begin{array}{l}\Delta \varepsilon \\ \Delta \gamma\end{array}\right\}$

trong đó ma trận $\left[\mathrm{D}_{\mathrm{se}}\right]$ được xác định theo biểu thức: $\left[\mathrm{D}_{\mathrm{se}}\right]=\left[\begin{array}{cc}\mathrm{k}_{\eta} & 0 \\ 0 & \mathrm{k}_{\xi}\end{array}\right]$

với: $\mathrm{k}_{\eta}$ và $\mathrm{k}_{\xi}$ tương ứng là độ cứng pháp tuyến và độ cứng tiếp tuyến của phần tử. Các hệ số này có thể được xác định bằng thực nghiệm hoặc xác định thông qua các đặc trưng của nền $[1,3]: \mathrm{k}_{\eta}=\frac{E(1-v)}{(1+v)(1-2 v)} ; \mathrm{k}_{\xi}=\mathrm{G}=\frac{\mathrm{E}}{2(1+v)}$

với: $v$ và $E$ tương ứng là hệ số Poisson và mô đun Young của vật liệu nền.

Chuyển vị một điểm bất kỳ của phần tử trong hệ trục toạ độ địa phương được xác định thông qua chuyển vị của nó trong hệ toạ độ tổng thể và góc lệch trục $\alpha$ :

$$
\left\{\begin{array}{l}
\mathrm{u}_{1} \\
\mathrm{v}_{1}
\end{array}\right\}=\left[\begin{array}{cc}
\cos \beta & \sin \beta \\
-\sin \beta & \cos \beta
\end{array}\right]\left\{\begin{array}{l}
\mathrm{u} \\
\mathrm{v}
\end{array}\right\}
$$

Ma trận độ cứng trong hệ toạ độ chung: $\left[\mathrm{K}_{\mathrm{se}}\right]=\iint\left[\mathrm{B}_{\mathrm{se}}\right]^{\mathrm{T}}\left[\mathrm{D}_{\mathrm{se}}\right]\left[\mathrm{B}_{\mathrm{se}}\right] \mathrm{dxdy}$

trong đó: $\left[\mathrm{B}_{\mathrm{se}}\right]=\left[\begin{array}{cc}\cos \beta & -\sin \beta \\ \sin \beta & \cos \beta\end{array}\right]\left[\mathrm{B}_{\text {local }}\right]$

Đối với PTTX 4 điểm nút, biến dạng trong phần tử là đều, ta có: 


$$
\left[\mathrm{B}_{\text {local }}\right]=\left[\begin{array}{cccccccc}
0 & \mathrm{~N}_{1}^{\prime} & 0 & \mathrm{~N}_{2}^{\prime} & 0 & -\mathrm{N}_{3}^{\prime} & 0 & -\mathrm{N}_{4}^{\prime} \\
\mathrm{N}_{1}^{\prime} & 0 & \mathrm{~N}_{2}^{\prime} & 0 & -\mathrm{N}_{3}^{\prime} & 0 & -\mathrm{N}_{4}^{\prime} & 0
\end{array}\right]
$$

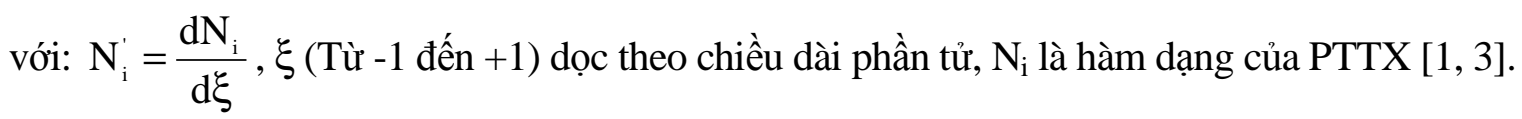

Sử dụng phép biến đổi $\iint \mathrm{dxdy}=\int_{-1}^{1} \operatorname{det}|\mathrm{J}| \mathrm{d} \xi$, ma trận độ cứng PTTX được xác định:

$$
\left[\mathrm{K}_{\mathrm{se}}\right]=\int_{-1}^{1}\left[\mathrm{~B}_{\mathrm{se}}\right]^{\mathrm{T}}\left[\mathrm{D}_{\mathrm{se}}\right]\left[\mathrm{B}_{\mathrm{se}}\right] \operatorname{det}|\mathrm{J}| \mathrm{d} \xi
$$

trong đó $[\mathrm{J}]$ là ma trận Jacobin $[1,3]$.

Ma trận độ cứng của PTTX trong công thức (15) được xác định bằng phương pháp tích phân số cầu phương Gauss-Legendre và sử dụng sơ đồ 1 điểm tích phân ( $\xi=0, \mathrm{w}=2)$.

\section{Phương trình chuyển động của kết cấu}

Khi động đất, gia tốc nền sẽ gây ra gia tốc chuyển vị của các khối lượng tập trung tại các nút của kết cấu $\left\{\ddot{\bar{U}}_{\mathrm{g}}\right\}$, được biểu diễn $[4,5,6,7]$ :

$$
\left\{\ddot{\mathrm{U}}_{\mathrm{g}}\right\}=\{\mathrm{r}\} \ddot{\mathrm{u}}_{\mathrm{g}} \text {. }
$$

trong đó: $\left\{\ddot{\bar{U}}_{\mathrm{g}}\right\}$ là véc tơ có số chiều bằng bằng số chiều véc tơ nút $\{\overline{\mathrm{U}}\}$ của hệ,

$\{r\}$ là véc tơ hệ số ảnh hưởng chuyển vị nút biểu thị chuyển vị tại các nút do chuyển vị ngang của nền bằng đơn vị gây ra, cấu trúc véc tơ gồm các phần tử 1 và 0 - tuỳ vào bậc tự do của mỗi nút và số chiều của gia tốc nền.

Gọi $\left\{\overline{\mathrm{R}}_{\mathrm{e}}\right\}$ là véc tơ tải trọng do động đất tác dụng lên các nút (là lực quán tính do gia tốc nền gây ra đối với đối với các khối lượng quy nút) của hệ. Lúc này ta có:

$$
\left\{\overline{\mathrm{R}}_{\mathrm{e}}\right\}=-[\overline{\mathrm{M}}]\left[\ddot{\overline{\mathrm{U}}}_{\mathrm{g}}\right\}=-[\overline{\mathrm{M}}]\{\mathrm{r}\} \ddot{\mathrm{u}}_{\mathrm{g}} .
$$

Phương trình chuyển động của hệ kết cấu chịu tác dụng đồng thời của tải trọng đã cho và động đất, có dạng $[4,5,6,7]$ :

$$
[\overline{\mathrm{M}}][\ddot{\overline{\mathrm{U}}}\}+[\overline{\mathrm{C}}][\dot{\mathrm{U}}\}+[\overline{\mathrm{K}}]\{\overline{\mathrm{U}}\}=\left\{\overline{\mathrm{R}}_{\mathrm{d}}\right\}+\left\{\overline{\mathrm{R}}_{\mathrm{e}}\right\}
$$

trong đó: $\left\{\overline{\mathrm{R}}_{\mathrm{d}}\right\}$ là véc tơ tải trọng nút do tải trọng đã cho gây ra. 


\section{THUẬT TOÁN GIẢI PHƯƠNG TRÌNH CHUYỂN ĐộNG CỦA KẾT CÂU}

Sau khi đưa vào các điều kiện biên, (18) có dạng tổng quát như sau [2, 4, 5, 6]:

$$
[M]\{\ddot{U}\}+[C]\{\dot{U}\}+[K]\{U\}=\left\{R_{d}\right\}+\left\{R_{e}\right\},
$$

Như đã biết, do kể đến sự tách, trượt cục bộ giữa kết cấu và nền san hô, phương trình chuyển động (19) là phương trình phi tuyến hình học và được viết lại như sau:

$$
[M][\ddot{U}\}+[C(\{\mathrm{U}\})]\{\dot{U}\}+[K(\{\mathrm{U}\})][\mathrm{U}\}=\left\{\mathrm{R}_{\mathrm{d}}\right\}+\left\{\mathrm{R}_{\mathrm{e}}\right\}=\{\mathrm{R}\}
$$

Ma trận cản tổng thể được xác định:

$$
[\mathrm{C}]=\alpha[\mathrm{M}]+\beta[\mathrm{K}]=[\mathrm{C}(\{\mathrm{U}\})] \text {, với: } \beta=\frac{2 \xi}{\omega_{1}+\omega_{2}} ; \alpha=\beta \omega_{1} \omega_{2} ;\left(\omega_{1}, \omega_{2} \text { là } 2\right. \text { tần số đầu tiên }
$$
trong dao động tự do, $\xi$ là tỷ số cản).

Để giải phương trình chuyển động (20) tác giả sử dụng phương pháp tích phân trực tiếp Newmark kết hợp với phương pháp lặp Newton - Raphson, với các bước tóm tắt thuật giải như trong bảng 2 .

Bảng 2: Tóm tắt thuật toán giải bài toán tương tác kết cấu công trình và nền san hô chịu tác dụng động đất theo phương pháp Newmark kết hợp với lặp Newton-Raphson

\section{Nhập dữ liệu ban đầu:}

1. Sơ đồ, kích thước kết cấu

2. Đặc trưng vật liệu

3. Nhập tải trọng (nếu có)

4. Nhập giản đồ gia tốc nền $\ddot{u}_{\mathrm{g}}(\mathrm{t})$

\section{Tính toán sơ bộ ban đầu:}

1. Tính các ma trận cơ bản: $[\mathrm{K}],[\mathrm{M}],[\mathrm{C}]$, véc tơ ảnh hưởng $\{\mathrm{r}\}$.

Xác định bước tích phân $\Delta \mathrm{t}$ và các tham số tích phân $\alpha, \delta$. Tính các hệ số của phương pháp: $a_{0}, a_{1}, a_{2}, a_{3}, a_{4}, \ldots, a_{7}$.

Xác định các véc tơ điều kiện ban đầu: $\left\{\mathrm{U}_{0}\right\},\left\{\dot{\mathrm{U}}_{0}\right\},\left\{\ddot{\mathrm{U}}_{0}\right\}$.

Tính ma trận độ cứng hiệu quả $\left[\mathrm{K}^{*}\right]$. 
II. Tính lặp cho các bước tích phân tại các thời điểm: $t=\Delta t, t=2 \Delta t, t=3 \Delta t, \ldots$ theo các bước sau:

1. Tính véc tơ tải trọng hiệu quả $\left\{\mathrm{R}_{\mathrm{t}+\Delta \mathrm{t}}^{*}\right\}$ tại thời điểm $\mathrm{t}+\Delta \mathrm{t}$

2. Dùng phương pháp lặp xác định véc tơ chuyển vị nút $\left\{U_{t+\Delta t}\right\}$ tại thời điểm $t+\Delta t$ :

$$
\begin{aligned}
& {\left[\mathrm{K}_{\mathrm{t}+\Delta \mathrm{t}}^{*}\right]\{\Delta \mathrm{U}\}^{(\mathrm{i})}=\left\{\mathrm{R}_{\mathrm{t}+\Delta \mathrm{t}}^{*}\right\}^{(\mathrm{i}-1)}-\left\{\mathrm{F}_{\mathrm{t}+\Delta \mathrm{t}}\right\}^{(\mathrm{i}-1)},} \\
& \left\{\mathrm{U}_{\mathrm{t}+\Delta \mathrm{t}}\right\}^{(\mathrm{i})}=\left\{\mathrm{U}_{\mathrm{t}+\Delta \mathrm{t}}\right\}^{(\mathrm{i}-1)}+\{\Delta \mathrm{U}\}^{(\mathrm{i})},
\end{aligned}
$$

Kiểm tra điều kiện hội tụ: $\frac{\left\|\{\Delta \mathrm{U}\}^{(\mathrm{i})}\right\|}{\left\|\left\{\mathrm{U}_{\mathrm{t}+\Delta t}\right\}^{(\mathrm{i})}-\left\{\mathrm{U}_{\mathrm{t}}\right\}\right\|} \leq \varepsilon_{\mathrm{D}}$

3. Xác định các véc tơ gia tốc và vận tốc tại thời điểm $\mathrm{t}+\Delta \mathrm{t}$ :

$$
\begin{aligned}
& \left\{\ddot{U}_{t+\Delta t}\right\}=a_{0}\left(\left\{U_{t+\Delta t}\right\}-\left\{U_{t}\right\}\right)-a_{2}\left\{\dot{U}_{t}\right\}-a_{3}\left\{\ddot{U}_{t}\right\} \\
& \left\{\dot{U}_{t+\Delta t}\right\}=\left\{\dot{U}_{t}\right\}+a_{6}\left\{\ddot{U}_{t}\right\}+a_{7}\left\{\ddot{U}_{t+\Delta t}\right\}
\end{aligned}
$$

4. Tính ứng suất, biến dạng và nội lực tại thời điểm $\mathrm{t}+\Delta \mathrm{t}$.

5. Kiểm tra điều kiện tách, trượt, cập nhật ma trận phần tử.

6. Tăng bước thời gian $\mathrm{t}=\mathrm{t}+\Delta \mathrm{t}$ và tiến hành lặp lại từ bước 1 .

\section{Ví DỤ SỐ}

Trên cơ sở các thuật toán đã thiết lập, tác giả đã lập chương trình tính có tên là Eartquake_2010. Chương trình được viết bằng ngôn ngữ MATLAB chạy trên máy tính sử dụng hệ điều hành Windows xp.

Tính kết cấu công sự trong nền san hô chịu đồng thời của lực thuỷ tĩnh tác dụng trực tiếp lên kết cấu và tải trọng động đất theo phương ngang, giản đồ gia tốc nền như hình 5 .

Sơ đồ bài toán như hình 2 , với các số liệu cụ thể như sau:

- Lực thuỷ tĩnh: $\mathrm{p}_{0}(\mathrm{t})=\mathrm{p}_{0}\left(-\frac{\mathrm{y}}{\mathrm{H}}+\frac{1}{2}\right)$, với $\mathrm{p}_{0}=200 \mathrm{~N} / \mathrm{cm}^{2}$.

- Kích thước hình bao của kết cấu: chiều cao $\mathrm{H}=4 \mathrm{~m}$, bề rộng $\mathrm{B}=5 \mathrm{~m}$, chiều dày $\mathrm{t}$ = 0,5 m. Vật liệu có $\mathrm{E}=0,34 \times 10^{7} \mathrm{~N} / \mathrm{cm}^{2}, v=0,3, \rho=2,5 \times 10^{-3} \mathrm{~kg} / \mathrm{cm}^{3}$. 


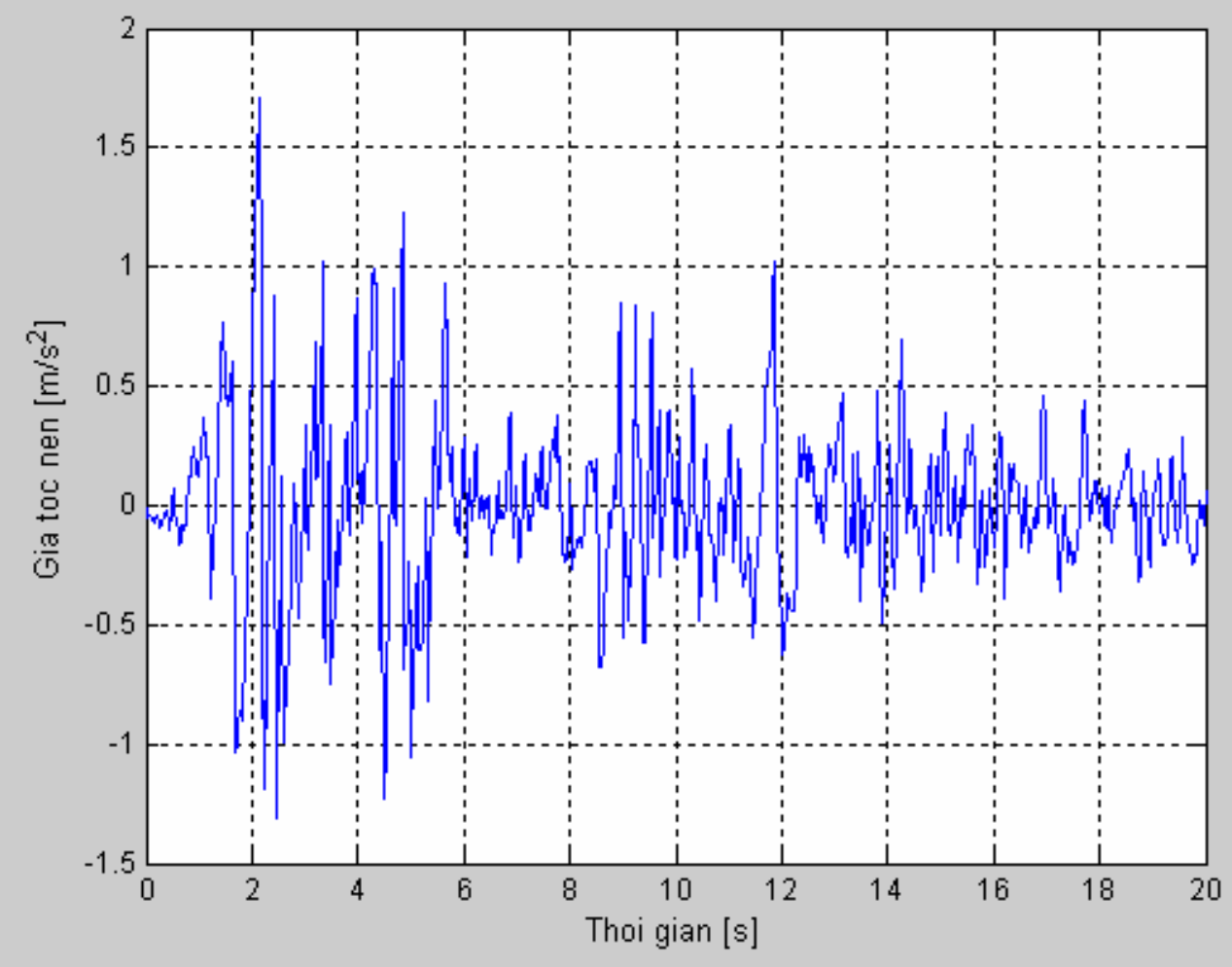

Hình 5: Biểu đồ gia tốc nền với giá trị cực đại $\mathrm{a}_{\max }=170,85 \mathrm{~cm} / \mathrm{s}^{2}[6]$

Sau khi lặp ứng với gia tốc nền lớn nhất, xác định được kích thước miền tính toán: $\mathrm{B}_{0}=20 \mathrm{~m}$, chiều cao $\mathrm{H}_{0}=14 \mathrm{~m}$, với sai số chuyển vị $\varepsilon=2,16 \%$, tương ứng với 5 lớp nền (thứ tự từ 1 đến 5 ) có đặc trưng cơ lý như bảng 3 .

Bảng 3: Thuộc tính vật liệu nền san hô [1]

\begin{tabular}{|c|c|c|c|c|c|c|}
\hline Lớp & $\begin{array}{c}\text { Độ sâu } \\
\text { (m) }\end{array}$ & $\begin{array}{c}E \\
\left(\mathrm{~N} / \mathrm{cm}^{2}\right)\end{array}$ & $v$ & $\begin{array}{c}\rho \\
\left(\mathrm{kg} / \mathrm{cm}^{3}\right)\end{array}$ & Hệ số ma sát f & $\begin{array}{c}\text { Hệ số giảm } \\
\text { chấn } \xi\end{array}$ \\
\hline 1 & 1 & $2,8.10^{4}$ & 0,22 & $2,5.10^{-3}$ & 0,21 & \multirow{5}{*}{0,05} \\
\hline 2 & 4 & $2,1.10^{5}$ & 0,25 & $2,8.10^{-3}$ & 0,32 & \\
\hline 3 & 8 & $2,0.10^{6}$ & 0,25 & $2,9.10^{-3}$ & 0,41 & \\
\hline 4 & 10 & $2,6.10^{5}$ & 0,25 & $2,0.10^{-3}$ & 0,47 & \\
\hline 5 & 14 & $2,3.10^{6}$ & 0,25 & $3,0.10^{-3}$ & 0,38 & \\
\hline
\end{tabular}

Mô hình PTHH của bài toán như hình 6. 


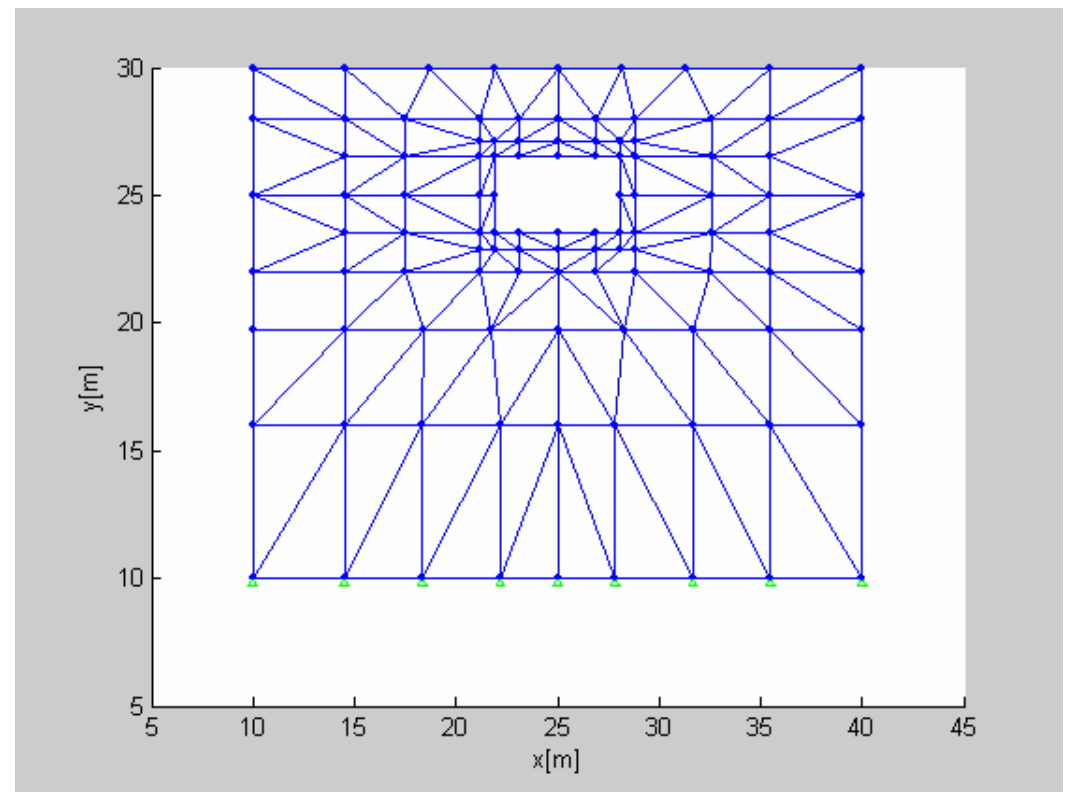

Hình 6: Mô hình PTHH của bài toán

Số lượng phần tử tổng cộng là 186 , bao gồm: 32 phần tử kết cấu, 20 phần tử tiếp xúc và 134 phần tử nền. Thời gian tính toán $\mathrm{t}_{\mathrm{cal}}=20 \mathrm{~s}$, bước thời gian $\Delta \mathrm{t}=0,02 \mathrm{~s}$.

\section{Kết quả tính:}

Hình 7 và 8 là ví dụ các kết quả đáp ứng chuyển vị ngang $U$ và ứng suất $\sigma_{\mathrm{x}}$ theo thời gian của điểm $\mathrm{C}$ (Điểm bên trái công sự).

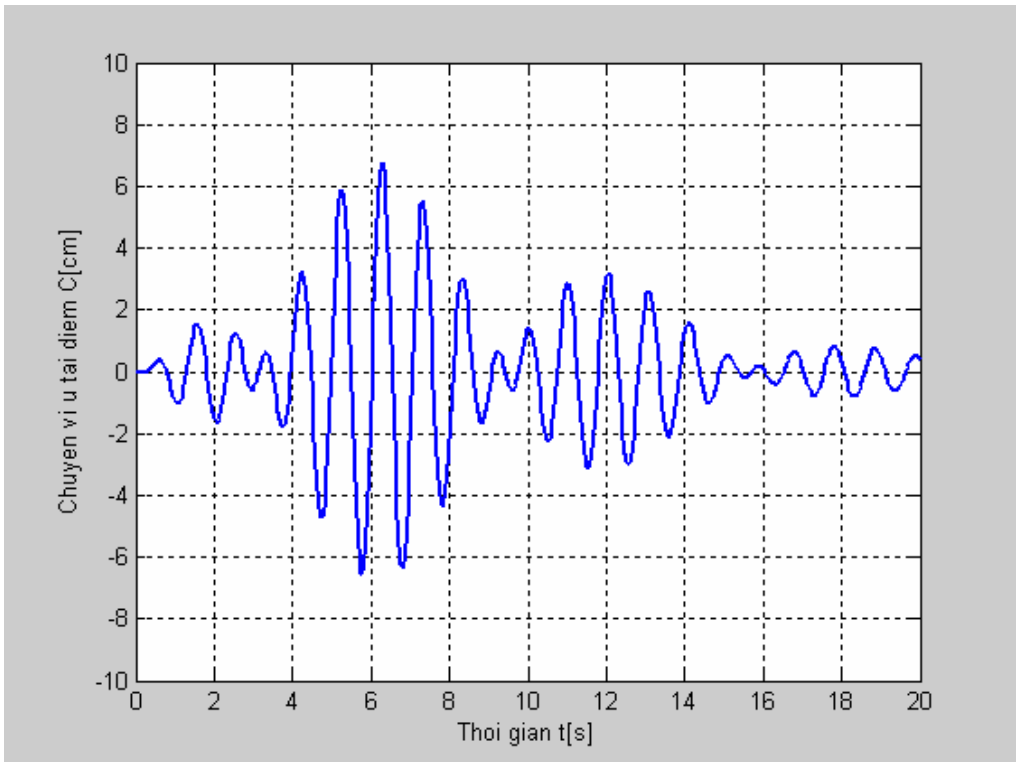

Hình 7: Sự thay đổi chuyển vị ngang tại điểm A thuộc đỉnh công sự 


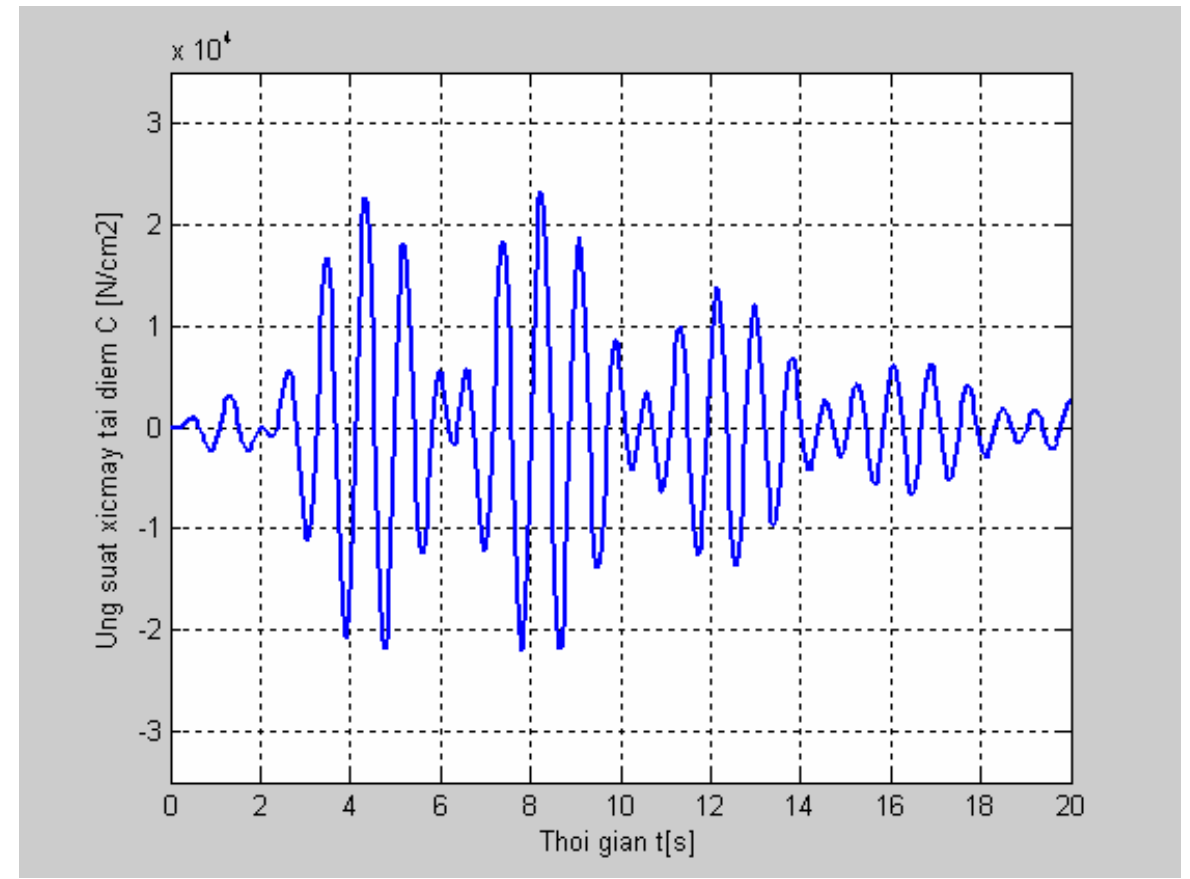

Hình 8: Sự thay đổi ứng suất $\sigma_{\mathrm{y}}$ tại điểm $\mathrm{C}$ thuộc đỉnh công sự

\section{KẾT LUẬN}

Nội dung bài báo đã đạt được:

- Xây dựng mô hình, thuật toán PTHH và chương trình phần mềm trong môi trường Matlab giải bài toán tương tác giữa kết cấu công trình và nền san hô chịu tác dụng đồng thời của lực thuỷ tĩnh và tải trọng do động đất gây nên.

- Tính toán trên ví dụ cụ thể cho thấy kết quả tính phù hợp quy luật cơ học.

- Kết quả bài báo có thể làm tài liệu tham khảo và là công cụ tính toán tương tác giữa kết công trình và nền san hô chịu tải trọng động đất phục vụ an ninh quốc phòng.

\section{TÀI LIỆU THAM KHẢO}

1. Nguyễn Thái Chung, 2006. Nền san hô và sự làm việc của cọc trong nền san hô, Luận án TSKT, Học viện KTQS.

2. Bathe K.J and Wilson E.L., 1978. Numerical Method in Finite Method Analyis Prentice, Hall of India Private Limited, New Delhi. 
3. Pizhong Qiao, Jialai Wang, 2004. Mechanics and fracture of crack tip deformable bi-material interface, International Journal of Solids and Structures 41 (2004) 7423 7444.

4. Giovanni Li Destri Nicosia, 2008. On seismic design and advanced numerical modelling of diaphragm walls under earthquake loading, Istituto Universittario di studi superiori di pavia universita degli studi di pavia.

5. Chen Guoxing và Zhuang Haiyang, 2009. Analysis on the erathquake response of subway station based on the substructuring suntraction method, Institute of geotechnical engineering, Nanjing university of technology, China.

6. Balideh S.,... 2009. Seismic analysis of underground spaces to propagation of seismic waves, Journal of applied sciences 9 (9), pp.1615-1627.

7. Jun Seong Choi, Jong Seh Lee, Jae Min Kim, 2009. Nonlinear earthquake respomse analysis of 2-D underground structures with soil-structures interaction including separation and sliding at interface, $15^{\text {th }} \mathrm{ASCE}$ engineering mechanics conference, Columbia University, New York.

\section{ANALYSIS OF UNDERGROUND STRUCTURE IN CORAL FOUNDATION UNDER EARTHQUAKE AND HYDROSTATIC LOAD}

\section{NGUYEN TAT NGAN}

Summary: In this study, the author present the calculating method interaction between underground structure and coral foundation under earthquake and hydrostatic load. The problem analysis with finite element method and using plane strain three nodes triangular element and 2D slip element (for slip layer between underground structure and coral foundation). The paper results can be used in design as reference for the structures in the coral foundation under earthquake loads.

Ngày nhận bài: 16 - 7 - 2010

Ngưòi nhận xét: PGS. TS. Phạm Tiến Đạt 